\title{
Game Theory Based Location Smoothing in Wireless Sensor Networks
}

\author{
http://dx.doi.org/10.3991/ijoe.v12i03.5400 \\ HE Yong-qiang ${ }^{1}$, QIN Qin ${ }^{1}$, WANG Jun-peng ${ }^{2}$ \\ ${ }^{1}$ Henan Institute of Engineering, Zhengzhou, China; \\ ${ }^{2}$ Beijing Institute of Technology, Beijing, China
}

\begin{abstract}
Location estimation of mobile target is an important research in wireless sensor networks. However, the phenomenon of non line of sight affects the accuracy of location estimating algorithms greatly. In order to improve the accuracy of location estimation of mobile targets, we proposed a game theory based location method algorithm in wireless sensor networks. In the proposed method, we first estimate the initial locations of mobile target with a support vector regression model, and then use game theory to smooth the already estimated locations. In the game model, game based filter and noise generator are two game rivals. The game filter aims to minimize the objective function of the game model, and the noise generator aims to maximize the same objective function. We propose a differential game theory based algorithm for solving the optimal resolution. The experiments show that, the proposed method is more powerful, and can be used to mobile target locating and tracking.
\end{abstract}

Index Terms-wireless sensor networks, location smoothing, game theory, non line of sight

\section{INTRODUCTION}

Nowadays, research of mobile location techniques [1] in wireless sensor networks is a hot research in both industry and academia. Mobile target locating and tracking is the basis on smart traffic [2], pervasive computing [3], location based service [4] and even the new internet of things [5]. The commonly used locating techniques include time of arrival (TOA), time difference of arrival (TDOA), angle of arrival (AOA), received signal strength indicator (RSSI), and so on. These traditional locating techniques are usually used in pervasive environment, but in wireless sensor networks, the factors of multiple paths, multiple addresses and especially the non line of sight (NLOS) bring much more error for the predicted location.

Mobile locating and tracking is actually the problem of location prediction, and NLOS is one of the most factors that bring errors [6]. Even we can apply some techniques to alleviate this kind of predicting error; the predicted location still deviates from the real trajectory of the target. So, in order to further smooth the predicted location trajectory for a mobile target, and get an accurate location prediction, filter techniques can be used after the initial location estimation. Traditional filter techniques include Kalman filtering [7], particle filtering [8] and so on, and these filtering methods need a priori knowledge of the target trajectory, and usually assume that the location error is a statistical stochastic process. However in practice, acquiring the statistical information or modeling the generation of errors, is difficult, and especially in the NLOS environment.

In this paper, we study the problem of location smoothing in wireless sensor networks. After initially estimating the locations of a mobile target, we propose to use game theory to further smooth the predicted locations of the mobile target. While solving the game based model, we propose a differential game theory based algorithm.

\section{RELATED WORKS}

In this section, we review related works of mobile target locating and tracking in wireless sensor networks, especially in the NLOS environment. Currently, the methods used to handle NLOS in wireless sensor networks can be classified into four types.

The first type is extracting line of sight. This kind of method applies the recognition mechanism of non line of sight, and uses the techniques, such as TDOA [9] and TOA [10], to classify the collected data. Then, the collected data are classified into signals of line of light and non line of sight [11]. The theoretical reason of this method is that, the variance of non line of sight is bigger than the variance of line of light. According to multiple measures, we can differentiate line of light from non line of sight. Theoretically, this method can be used to differentiate different signals, but when the contrast is small, i.e. in a pure non line of light environment, this method is not suitable [12].

The second type is reconstructing values of non line of sight. This method is usually applied in practice, and the main idea is that, get the NLOS values according to the TOA [13] and RSSI [14] methods and estimate the mean value and variance of delay and error based on the value distribution. By removing the non line of sight error from the measured values, the reconstructed values are an approximation of the measured values of line of sight. In this method, researchers must know the prior knowledge of the distribution, and otherwise, the error of non line of sight cannot be removed.

The third type is introducing the weights and the revised factors. This kind of method is very simple. After getting the line of sight and non line of sight signals via TOA [15] and TDOA [16], it weights these signals differently, where the weights of line of sight are far bigger than the weights of non line of sight. Then during the computing of locations, the error of location estimation can be reduced efficiently. However, the disadvantage of this method is also that the statistical features of stochastic noise must be already known. 
The last type of method to handle NLOS in wireless sensor networks is the hybrid method, and it applies TDOA/AOA [17] or TOA/AOA [18] to estimate the hybrid locations. This kind of method can improve the precision of estimated locations to a certain extent, but when the error of some measured value from one method exceeds the threshold, the result is still bad. In addition, the hybrid method needs to run multiple methods at the same time, and increases the time of location estimation greatly, so it is not suitable to the real-time wireless sensor networks.

Besides the above methods, Kalman filter [7], restrained non-linear optimal method [19] and Markov based pattern recognition [20] can also be used to location smoothing. However, these methods use the accurate values of arrival time, difference of arrival time and signal strength, and in the non line of sight environment, these values can't be measured directly, so they are also not suitable to the problem of non line of sight.

\section{SUPPORT Vector MACHINE BASED LOCATION ESTIMATING}

\section{A. Support vector machine}

Support vector machine (SVM) [21] is a kind data based learning method, and the main idea is to build a model from the data and apply the model to predict unknown data. SVM is a linear optimal classification hyperplane, and figure 1(a) is an illustration of SVM in a 2 dimensional plane. There are two types of samples, and $H$ is the optimal plane classifying them. The samples on lines of $H_{1}$ and $H_{-1}$ are support vectors.

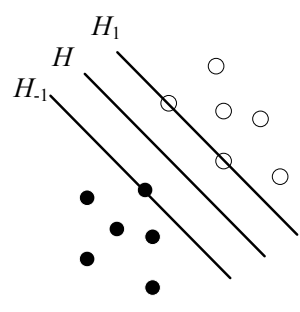

(a) Optimal classifier

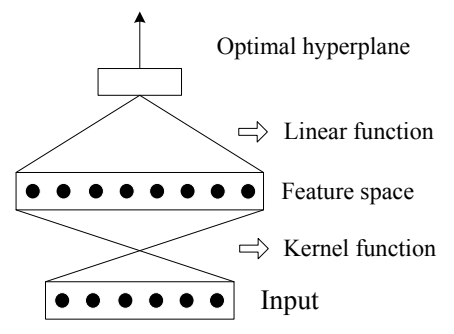

(b) Support vector regression
Figure 1. Support vector machine

In a high dimensional space, for samples of $\left(x_{i}, y_{i}\right)$ ( $i=1, \ldots, n$ ), the optimal hyperplane of SVM is

$$
w \Delta x+b=0
$$

Where $w \Delta x$ is the inner product of $w$ and $b$, and $w$ and $b$ are parameters that satisfy

$$
y_{i}\left(w \Delta x_{i}+b\right) \geq 1, i=1, \ldots, n .
$$

In order to get the values of $w^{\prime}=[w, b]$, we need to minimize the following object function

$$
f\left(w^{\prime}\right)=\frac{1}{2}\|w\|^{2} .
$$

For the samples that can be linearly classified, we can get the optimal classifier by the object function in equation 2 and constraints in equation 1. However, real samples usually cannot be linearly classified, and then we can map them to a higher dimensional space, where they can be linearly classified. Figure 2(b) is a typical SVM that can handle linearly unclassified samples. By using kernel function, we can map the original input data to a higher dimensional space, and then use a linear function to classify them.

Kernel function is the basis of SVM, and it transforms the linearly unclassified problem into a linearly classified problem. In figure 2, $x$ and $o$ are two types of samples in the original space, and we aim to classify them. Obviously, they cannot be classified linearly in the original space. However, we can map these points into a higher space via a kernel function $F(\diamond)$, such that they can be classified linearly in the higher dimensional space.

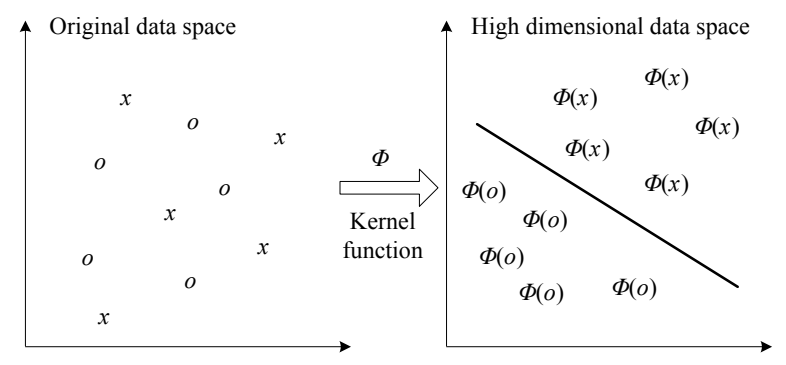

Figure 2. Illustration of Kernel function

In the Hilebert space, we use the following inner product formula

$$
\left(X_{i}, X\right)=K\left(x_{i}, x\right),
$$

Where $x$ is the vector in the original space, $X$ is the mapped vector in the high dimensional feature space, and $K\left(x_{i}, x\right)$ is the kernel function, which replaces the inner product in the high dimensional feature space. So, the object function in equation 3 can be represented as the following equation:

$$
\left\{\begin{array}{l}
\underset{a}{\arg \max } \sum_{i=1}^{n} a_{i}-\frac{1}{2} \sum_{i=1}^{n} \sum_{j=1}^{n} a_{i} a_{j} y_{i} y_{j} K\left(x_{i}, y_{j}\right) \\
0 \leq a_{i} \leq C, i=1, \ldots, n \\
\sum_{i=1}^{n} a_{i} y_{i}=0
\end{array}\right.
$$

\section{B. Location estimating algorithm}

In this paper, we don't use TOA, TDOA, AOA and RSSI as the metrics for distance, and on the contrast, we use them as features of location. So, we have a number of real locations and their corresponding features. We use the SVM to build a relationship between the real locations and the features, and use the SVM regression model to predict future locations while their features are given.

In order to describe the relationship between input and output, the training data are $\operatorname{Tr}=\left\{\left(m_{i}, p_{i}\right) \mid 1 £ i £ l\right\}$, where $m_{i} \mathrm{OE} R^{n}$ is a $n$ dimensional feature vector and $p_{i}$ is a location point. Because the relationship between locations with features is not linear, we use the support vector machine to map the feature vector to a high dimensional space, such that the high dimensional vectors and the locations have a linear relationship. Simply, we map $m$ to a high dimensional space via the kernel function $F(m)$, and then the linear regression function is

$$
p=f(m)=F(m)^{T} \Delta w+b,
$$


where $F: R^{n} Æ F, w \mathrm{CEF}$, and $F$ is the high dimensional space.

The above support vector regression model estimates the parameter $w$ by minimizing the following empirical risk function

$$
R(w)=\sum_{i=1}^{l} g\left(f\left(m_{i}\right)-p_{i}\right)+\frac{1}{2 c}\|w\|^{2},
$$

where $g(\cdot)$ is the cost function, and defined as follows:

$$
g(f(m)-p)=\left\{\begin{array}{cc}
|f(m)-p|, & \text { if }|f(m)-p|>\varepsilon, \\
0, & \text { otherwise. }
\end{array},\right.
$$

Where $e$ is a predefined hyper parameter. When the difference between the observed value with the predicted value is less than $e$, we assume that they are the same.

According to the Lagrane method, we can represent the above problem with its dual problem, and then the optimal object function based on support vector regression is

$$
\begin{gathered}
\underset{a, a^{\prime}}{\arg \max } \sum_{i=1}^{l}\left[a_{i}^{\prime}\left(p_{i}-\varepsilon\right)-a_{i}^{\prime}\left(p_{i}+\varepsilon\right)\right]- \\
\frac{1}{2} \sum_{i=1}^{l} \sum_{j=1}^{l}\left(a_{i}^{\prime}-a_{i}\right)\left(a_{j}^{\prime}-a_{j}\right) K\left(m_{i}, m_{j}\right),
\end{gathered}
$$

where the kernel function $K\left(m_{i}, m_{j}\right)=F\left(m_{i}\right)^{T} F\left(m_{j}\right)$, $a_{i}, a_{i}^{\prime}$ are the Lagrane multipliers, and the constraints are

$$
\begin{gathered}
\sum_{i=1}^{l}\left(a_{i}-a_{i}^{\prime}\right)=0,0 \leq a_{i}, a_{i}^{\prime} \leq C, \text { and } \\
w=\sum_{i=1}^{l}\left(a_{i}-a_{i}^{\prime}\right) \Phi\left(m_{i}\right) .
\end{gathered}
$$

Finally, we can get the regression function as follows:

$$
p=f(m)=\sum_{i=1}^{l}\left(a_{i}-a_{i}^{\prime}\right) K\left(m_{i}, m\right)+b .
$$

\section{GAME THEORY BASED LOCATION SMOOTHING}

\section{A. Problem model}

During locating and tracking of a target, the measured values of sensors are usually contaminated by noise. The noise cannot be avoided, but we can try our best to reduce them. The values measured by sensors include the distances between the target with anchor points and the angles, and these values are usually contaminated by the Gaussian or non-Gaussian noise, which results in the deviated values.

In this section, we aim to smooth the predicted location of a target, and make the predicted location more accurate. After estimating the locations of a target via SVM regression, the estimated values still deviate from their real values. However for a series of predicted locations, we can further smooth these predicted values, and thus get more accurate location estimation.

We assume that the distribution of noise is unknown, and use the game theory to smooth the predicted locations. This step follows the SVM regression step. In the SVM regression step, we measure the feature vector of the target every $\mathrm{D} T$ time, let the estimated value at time $t$ be $y^{\prime}(t)=\left[y_{1}^{\prime}(t), y_{2}^{\prime}(t)\right]^{T}$, and then the feature vector is $x^{\prime}\left[x_{1}^{\prime}, x_{2}^{\prime}, x_{3}^{\prime}, x_{4}^{\prime}\right]^{T}$, where $x_{1}^{\prime}, x_{2}^{\prime}$ are the state coordinate, and $x_{3}^{\prime}, x_{4}^{\prime}$ are the speed coordinate. Then at time $k \mathrm{D} T$, the state element is computed as follows

$$
x_{k+1}^{\prime}=A^{\prime} x_{k}^{\prime}+O u_{k}+B w_{k}+d_{k} \text {. }
$$

where $k$ is the time index, $x$ is the state vector, $u_{k}$ is the 2 dimensional speedup, $w_{k}$ and $n_{k}$ are while noises, $d_{k}$ is the noise sequence generated by the game rival. Then, the relationship between estimated locations with states is

$$
y_{k}^{\prime}=H^{\prime} x_{k}^{\prime}+n_{k} \text {. }
$$

In equations 13 and 14 , parameters $A^{\prime}, O$ and $B$ are $A^{\prime}=\left[\begin{array}{cccc}1 & 0 & \Delta T & 0 \\ 0 & 1 & 0 & \Delta T \\ 0 & 0 & 1 & 0 \\ 0 & 0 & 0 & 1\end{array}\right] \quad, \quad O=\left[\begin{array}{cc}0 & 0 \\ 0 & 0 \\ \Delta T & 0 \\ 0 & \Delta T\end{array}\right]$ $H^{\prime}=\left[\begin{array}{ll}1 & 0 \\ 0 & 1 \\ 0 & 0 \\ 0 & 0\end{array}\right]$.

Let $x_{k}=\left[x_{k}^{\prime}, u_{k}\right]^{T}$ and $y_{k}=y_{k}^{\prime}$, then equations 13 and 14 can be rewritten to

$$
\begin{gathered}
x_{k+1}=A x_{k}+B w_{k}+d_{k}, \text { and } \\
y_{k}=H x_{k}+n_{k},
\end{gathered}
$$

Where $A=\left[A^{\prime}, O\right]$, and $H=\left[H^{\prime}, O\right]$.

Now, in order to predict the locations of $\left\{y_{1}, \ldots, y_{k}\right\}$, we need to find the estimation, $\hat{x}_{k+1}$, for $x_{k+1}$. If the estimation is unbiased, then we have

$$
\begin{gathered}
\hat{x}_{0}=0, \\
\hat{x}_{k+1}=A \hat{x}_{k}+K_{k}\left(y_{k}-H_{k} \hat{x}_{k}\right) .
\end{gathered}
$$

In equation $13, d_{k}$ is the noise sequence generated by the game rival, and according to the game theory, its aim is to maximize the estimation error of locations, so we can assume the input system of the game rival is

$$
d_{k}=L_{k}\left(G_{k}\left(x_{k}-\hat{x}_{k}\right)+n_{k}\right),
$$

where $L_{k}$ is the gain to be determined, $G_{k}$ is the predefined matrix, and $n_{k}$ is the noise sequence. At this moment, $n_{k}$ and $w_{k}$ are two independent noise sequences, and they are both irrelevant to $x_{0}$.

The estimated error is $e_{k}=x_{k}-\hat{x}_{k}$, and as the time goes on, the dynamical estimation equation of errors are

$$
e_{0}=x_{0},
$$

$e_{k+1}=\left(A-K_{k} H+L_{k} G_{k}\right) e_{k}+B w_{k}+L_{k} n_{k}-K_{k} n_{k}$

If the game rival increases $L_{k}$, then the noise $e_{k}$ can be arbitrarily large, so the minimax problem in this game is biased. In order to prevent this problem, $e_{k}$ can be divided into two parts, $e_{k}=e_{1, k}+e_{2, k}$, and then their dynamical estimation equation for errors are 


$$
\begin{gathered}
e_{0}=x_{0}, \\
e_{1, k+1}=\left(A-K_{k} H+L_{k} G_{k}\right) e_{1, k}+B w_{k}-K_{k} n_{k}, \\
e_{2, k+1}=\left(A-K_{k} H+L_{k} G_{k}\right) e_{2, k}+L_{k} n_{k} .
\end{gathered}
$$

According to game theory, we construct two rivals, and they location estimation and state noise. Then, the object function becomes

$$
J\left(K_{k}, L_{k}\right)=\operatorname{tr} \sum_{k=1}^{N} w_{k} E\left(e_{1, k} e_{1, k}^{T}-e_{2, k} e_{2, k}^{T}\right),
$$

Where $w_{k}$ is a positive definite matrix.

The main idea of game based filter is that, the location estimation aims to reduce $J$ by finding a gain sequence, and its rival aims to increase $J$ by finding a gain sequence. Then, upon $J, L_{k}$ and $K_{k}$, a tracking model is built based on the game theory.

\section{B. Location smoothing algorithm}

According to the differential game theory [22], the optimal resolutions of gain sequence in an objective function are the saddle points $\left(K_{k}^{*}, L_{k}^{*}\right)$. That is, for the optimal resolution $\left(K_{k}, L_{k}\right)$, it must satisfies that $J\left(K_{k}^{*}, L_{k}\right) £ J\left(K_{k}^{*}, L_{k}^{*}\right) £ J\left(K_{k}, L_{k}^{*}\right)$.

In order to solve the above problem, we can simplify the objective function as follows:

$$
\begin{gathered}
F_{k}=A-K_{k} H+L_{k} G_{k}, \\
Q_{0}=E\left(x_{0} x_{0}^{T}\right), \\
Q_{k+1}=F_{k} Q_{k} F_{k}^{T}+B B^{T}+K_{k} K_{k}^{T}-L_{k} L_{k}^{T} .
\end{gathered}
$$

Then, the objective function becomes

$$
J\left(K_{k}, L_{k}\right)=\operatorname{tr} \sum_{k=1}^{N} w_{k} Q_{k} .
$$

Assuming that $\bar{Q}_{0}$ and $S_{k}$ are the nonsingular solutions of the following equations:

$$
\begin{gathered}
\bar{Q}_{0}=E\left(x_{0} x_{0}^{T}\right), \\
\bar{Q}_{0}\left(I-H^{T} H S_{k}\right)=\left(I-\bar{Q}_{k} G_{k}^{T} G_{k}\right) S_{k}, \\
\bar{Q}_{k+1}=A S_{k} A^{T}+B B^{T} .
\end{gathered}
$$

In a game model, the nonsingular solution can't be sure to exist, but once a nonsingular solution exists, it must have

$$
S_{k}=\left(\bar{Q}_{k} H^{T} H-\bar{Q}_{k} G_{k}^{T} G_{k}+I\right)^{-1} \bar{Q}_{k} .
$$

According to reference [22], if $H \bar{Q}_{k} H^{T}+I \geq 0$ and $I-G_{k} \bar{Q}_{k} G_{k}^{T} \geq 0$, then $L_{k}^{*}=A S_{k} G_{k}^{T}, K_{k}^{*}=A S_{k} H^{T}$ satisfy the saddle conditions.

Finally, the game theory based filter algorithm is:

1) Define a linear system

$$
\begin{gathered}
x_{k+1}=A x_{k}+B w_{k}+d_{k} \\
y_{k}=H x_{k}+n_{k}
\end{gathered}
$$

where $n_{k}$ and $w_{k}$ are two independent noise sequences, and $d_{k}$ is the noise sequence generated by the game rival.

2) Initialize

$$
\hat{x}_{0}=0, \hat{Q}_{0}=E\left(x_{0} x_{0}^{T}\right)^{T}
$$

3) For $k=0,1, \ldots$, iterate the following steps:

a) Select the parameter $G_{k}$, and its value is determined by the input noise generated by the game rival;

b) Computing the following estimations:

$$
\begin{gathered}
S_{k}=\left(\bar{Q}_{k} H^{T} H-\bar{Q}_{k} G_{k}^{T} G_{k}+I\right)^{-1} \bar{Q}_{k} \\
\bar{Q}_{k+1}=A S_{k} A^{T}+B B^{T} \\
K_{k}=A S_{k} H^{T} \\
\hat{x}_{k+1}=A \hat{x}_{k}+K_{k}\left(y_{k}-H_{k} \hat{x}_{k}\right)
\end{gathered}
$$

c) Validate $I-\bar{Q}_{k} G_{k}^{T} G_{k} \geq 0$. if it doesn't satisfy, then reselect $G_{k}$ by reducing it and go to step 1 .

\section{EXPERIMENTS}

In the experiments, we simulate a $600 \mathrm{~m} \times 600 \mathrm{~m}$ region, and there are three anchor points placed in the region. The initial speed of the simulated target is $10 \mathrm{~m} / \mathrm{s}$, and we measure the TOA features of the target five times per second.

Firstly, we compare the mean errors of four locating algorithms by changing the value of $\alpha$, and the result is in figure 3 . Here, we implement the least square algorithm, support vector regression, the combination of support vector regression and Kalman filter and the combination of support vector regression and game theory. In these algorithms, least square and support vector regression are used to estimate the locations of the target, and Kalman filter and game theory are used to smooth the tracking. As we can see from the figure that, the mean error of the least square algorithm increases greatly when $\alpha$ increases; the mean errors of SVR, SVR+Kalman and SVR+Game increase gently; and SVR+Game has the lowest mean error in these four algorithms.

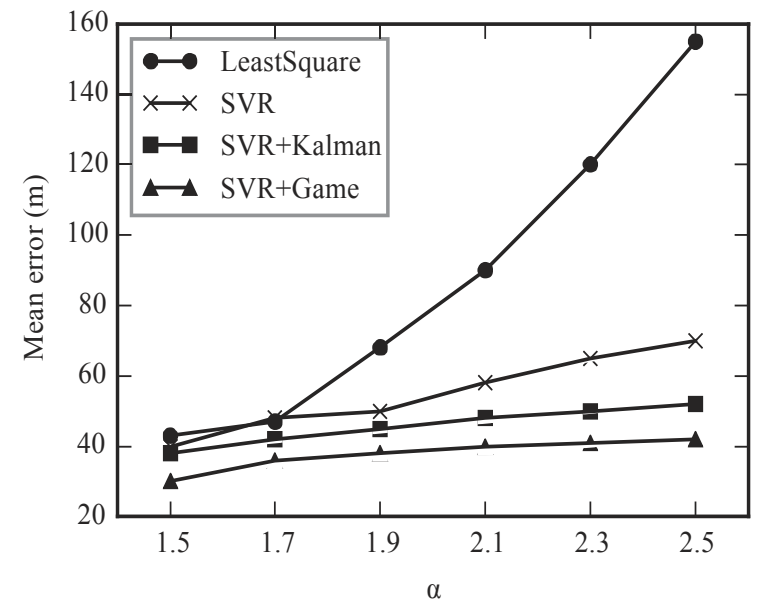

Figure 3. Comparison of mean error

Next, we compare the running time of the above algorithms for estimating locations of the target. In figure 4, as the SVR+kalman and SVR+Game algorithms add an additional smoothing process after the initial location estimation (SVR) process, the running time is longer. However, the adding of running time brings the improvement of location estimation (figure 3), and the adding is tolerant in an embedded system, so smoothing 
techniques are useful in mobile location estimating. In addition, the additional Game step is much more efficient than the Kalman filter when smoothing location estimation values.

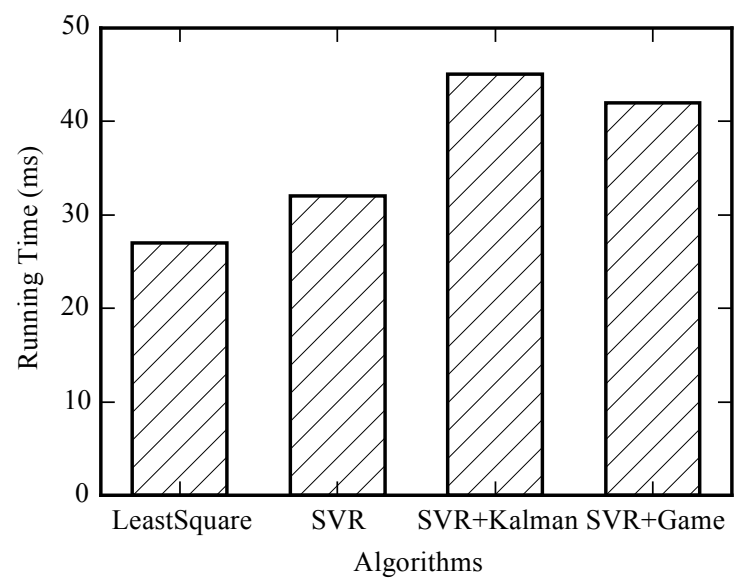

Figure 4. Comparison of running time

Finally, we compare the locating effectiveness of SVR+Kalman and SVR+Game via two samples. Figure 5 illustrates the results of the location estimation via the SVR+Kalman algorithm, and figure 6 is the results via the SVR+Game algorithm. By comparing these two figures, we can see that the SVR+Game algorithm is more effective than the SVR+Kalman algorithm while predicting the locations of the target. So, we can conclude that the proposed game theory based smoothing technique is more powerful, and can be used to mobile locating and tracking of target.

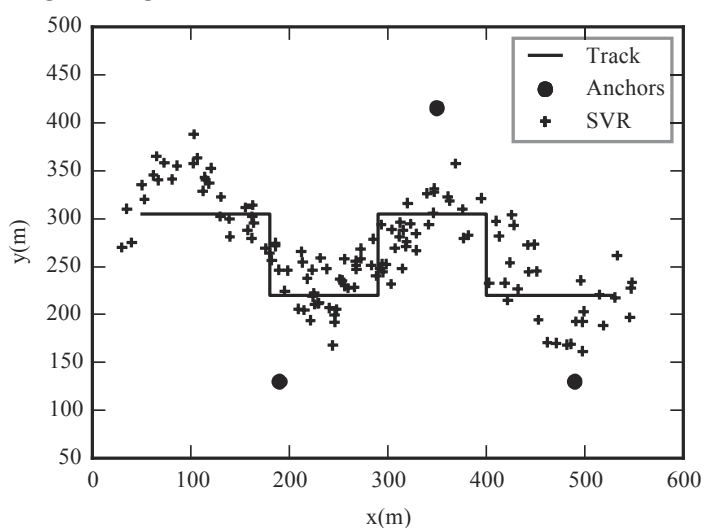

Figure 5. Illustration of location estimation in SVR+Kalman

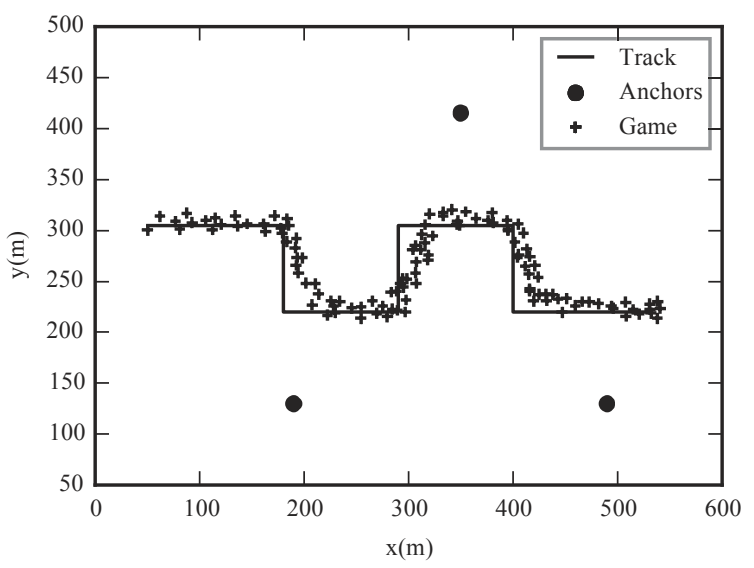

Figure 6. Illustration of location estimation in SVR+Game

\section{CONCLUSION}

This paper studies the problem of location smoothing in mobile target locating and tracking, and proposes a game theory based location smoothing method. The proposed method is an additional step after the initial location estimation based on the support vector regression. In the game based model, game based filter and noise generator are two game rivals. The game filter aims to minimize the objective function of the game model, and the noise generator aims to maximize the same objective function. We propose a differential game theory based algorithm for solving the optimal resolution. The experiments show that, the proposed method is more powerful, and can be used to mobile target locating and tracking.

\section{REFERENCES}

[1] Zeimpekis V, Giaglis G M, Lekakos G. A taxonomy of indoor and outdoor positioning techniques for mobile location services[J]. ACM SIGecom Exchanges, 2002, 3(4): 19-27. http://dx.doi.org/10.1145/844351.844355

[2] Bottigliengo M, Casetti C, Chiasserini C F, et al. Smart traffic scheduling in 802.11 WLANs with access point[C]//Vehicular Technology Conference, 2003. VTC 2003-Fall. 2003 IEEE 58th. IEEE, 2003, 4: 2227-2231.

[3] Satyanarayanan M. Pervasive computing: Vision and challenges[J]. Personal Communications, IEEE, 2001, 8(4): 10-17. http://dx.doi.org/10.1109/98.943998

[4] Liu D, Ning P. Location-based pairwise key establishments for static sensor networks[C]/Proceedings of the 1st ACM workshop on Security of ad hoc and sensor networks. ACM, 2003: 72-82.

[5] Jin Z, Shi D, Wu Q, et al. Random Walk Based Location Prediction in Wireless Sensor Networks[J]. International Journal of Distributed Sensor Networks, 2013, 2013.

[6] Borras J, Hatrack P, Mandayam N B. Decision theoretic framework for NLOS identification[C]//Vehicular Technology Conference, 1998. VTC 98. 48th IEEE. IEEE, 1998, 2: 15831587.

[7] Long Le B, Ahmed K, Tsuji H. Mobile location estimator with NLOS mitigation using Kalman filtering[C]//Wireless Communications and Networking, 2003. WCNC 2003. 2003 IEEE. IEEE, 2003, 3: 1969-1973.

[8] Hightower J, Borriello G. Particle filters for location estimation in ubiquitous computing: A case study[M]//UbiComp 2004: Ubiquitous Computing. Springer Berlin Heidelberg, 2004: 88-106.

[9] Gholami M R, Gezici S, Ström E G. Improved position estimation using hybrid TW-TOA and TDOA in cooperative networks[J]. Signal Processing, IEEE Transactions on, 2012, 60(7): 3770-3785. http://dx.doi.org/10.1109/TSP.2012.2194705

[10] Geng Y, He J, Deng H, et al. Modeling the effect of human body on TOA ranging for indoor human tracking with wrist mounted sensor $[\mathrm{C}] / /$ Wireless Personal Multimedia Communications (WPMC), 2013 16th International Symposium on. IEEE, 2013: 1-

[11] Chen H, Wang G, Wang Z, et al. Non-line-of-sight node localization based on semi-definite programming in wireless sensor networks[J]. Wireless Communications, IEEE Transactions on, 2012, 11(1): 108-116. http://dx.doi.org/10.1109/TWC.2011. 110811.101739

[12] Groves P D. GNSS Solutions: Multipath vs. NLOS signals. How Does Non-Line-of-Sight Reception Differ From Multipath Interference[J]. Inside GNSS Magazine, 2013, 8(6): 40-42.

[13] Shen J, Molisch A F, Salmi J. Accurate passive location estimation using TOA measurements[J]. Wireless Communications, IEEE Transactions on, 2012, 11(6): 2182-2192 http://dx.doi.org/10.1109/TWC.2012.040412.110697.

[14] Luo X, O'Brien W J, Julien C L. Comparative evaluation of Received Signal-Strength Index (RSSI) based indoor localization techniques for construction jobsites[J]. Advanced Engineering Informatics, 2011, 25(2): 355-363. http://dx.doi.org/10.1016/j.aei. 2010.09.003 
PAPER

GAME THEORY BASEd LOCATION SMOOTHING IN WIRELESS SENSOR NETWORKS

[15] Liu W, Ding H, Huang X, et al. TOA estimation in IR UWB ranging with energy detection receiver using received signal characteristics[J]. Communications Letters, IEEE, 2012, 16(5): 738-741. http://dx.doi.org/10.1109/LCOMM.2012.030912.112445

[16] Yu H, Huang G, Gao J, et al. An efficient constrained weighted least squares algorithm for moving source location using TDOA and FDOA measurements[J]. Wireless Communications, IEEE Transactions on, 2012, 11(1): 44-47. http://dx.doi.org/10.1109/ TWC.2011.102611.110728

[17] Lim D W, Kang J M, Heo M B. Hybrid TDOA/AOA localization algorithm for GPS jamming[J]. Journal of Institute of Control, Robotics and Systems (in Korean), 2014, 20(1): 101-105. http://dx.doi.org/10.5302/J.ICROS.2014.13.1943

[18] Ding G, Tan Z, Zhang L, et al. Hybrid TOA/AOA cooperative localization in non-line-of-sight environments[C]//Vehicular Technology Conference (VTC Spring), 2012 IEEE 75th. IEEE, 2012: 1-5.

[19] Dong G, Ma Z D, Hulbert G, et al. An efficient optimal design methodology for non-linear multibody dynamics systems with application to vehicle occupant restraint systems[J]. International Journal of Vehicle Design, 2012, 61(1-4): 177-203.

[20] Mathew W, Raposo R, Martins B. Predicting future locations with hidden Markov models[C]//Proceedings of the $2012 \mathrm{ACM}$ conference on ubiquitous computing. ACM, 2012: 911-918.

[21] Golparvar-Fard M, Heydarian A, Niebles J C. Vision-based action recognition of earthmoving equipment using spatio-temporal features and support vector machine classifiers[J]. Advanced Engineering Informatics, 2013, 27(4): 652-663. http://dx.doi.org/10.1016/j.aei.2013.09.001

[22] Buckdahn R, Cardaliaguet P, Quincampoix M. Some recent aspects of differential game theory[J]. Dynamic Games and Applications, 2011, 1(1): 74-114. http://dx.doi.org/10.1007/ s13235-010-0005-0

\section{AUTHORS}

He Yong-qiang is with the College of Computer, Henan Institute of Engineering, Zhengzhou, China. (email: heyongqiangxz@163.com).

Qin Qin is with the College of Computer, Henan Institute of Engineering, Zhengzhou, China.

WANG Jun-peng is with the School of Computer Science \& Technology, Beijing Institute of Technology, Beijing, China.

This work was supported in part by the Project of science and technology department of Henan province (NO.152102210027) and the Project of education department of Henan province (NO.15A520054). Submitted 22 December 2015. Published as resubmitted by the authors 21 January 2016. 